\title{
Issues and Applications of Real-Time Data from Off-Grid Electrical Systems
}

\author{
Henry Louie, George Goldsmith \\ Seattle University, \\ Seattle, WA, USA \\ louieh@seattleu.edu, \\ goldsmi1@seattleu.edu
}

\author{
Peter Dauenhauer \\ University of Strathclyde \\ Glasgow, United Kingdom \\ Peter.dauenhauer@strath.ac.uk
}

\author{
Rita H. Almeida \\ University of Lisbon \\ Lisbon, Portugal \\ rhalmeida@fc.ul.pt
}

\begin{abstract}
Off-grid systems are a pathway to electrification for millions of people located far from the existing grid. However, the premature failure and underutilization of these systems are difficult to prevent. Advances in data acquisition hardware, the expansion of cellular communication networks and cloud-based solutions have made data collection, monitoring, archival and even remote interaction with off-grid systems a possibility. The co-installation of data acquisition hardware with off-grid systems is not a wide-spread practice, and use cases of the data have not been popularized. This paper discusses the technology, applications and considerations related to off-grid data acquisition and monitoring. Data from several off-grid systems in Sub-Saharan Africa are used to illustrate the applications.
\end{abstract}

Keywords-Data acquisition; energy kiosk; energy poverty; microgrids; rural electrification

\section{INTRODUCTION}

The International Energy Agency expects one-half of new electrical connections in less economically developed countries (LEDCs) to be provided by mini- or micro-grids [1]. The longterm viability of these systems to provide life-changing electricity is tenuous and dependent on many factors [2]. Micro-grids and mini-grids in LEDCs - hereafter collectively referred to as "microgrids"-face numerous technical challenges. Microgrids are often installed in remote locations. As a consequence, routine maintenance and repair trips can be costly and inconvenient, and may be put-off or postponed to the detriment of the system. Reliance of some microgrids on stochastic sources such as photovoltaic panels and wind turbines necessitate the use of batteries and also complicate the energy management of the system. Improper controller setpoints or missoperation of the system can accelerate failure modes. The demand itself is also difficult to estimate a priori, and so the microgrid might not be appropriately designed to begin with [3]-[5], leading to low reliability or, conversely, wasted excess capacity.

The technical challenges can be mitigated to some extent by installing real-time data acquisition, broadcast, monitoring and actuation systems, hereafter referred to as "Remote Monitoring" (RM) systems. RM systems measure quantities such as battery voltage, source current, energy consumption and other analog or digital signals. The measurements are broadcast through the growing rural cellular communication network to a server where they can be used for analysis or archived for later use.

The co-installation of RM systems with microgrids is becoming increasingly common, although many new systems are installed without this capability. The best-practices of RM system design, and use-cases of the data are not well-known or wide-spread. The use of microgrid data in the literature is scant [6,7], but the need for it is well-known [3,4]. To address the gap in literature and practice, this paper describes the issues of RM systems and the application of the data they provide to improve the operation and technical sustainability of microgrids. The paper draws upon data sets from microgrids in Kenya and Zambia to illustrate applications of data.

The remainder of this paper is arranged as follows. Section II provides an overview of RM systems. Practical considerations of RM systems are discussed in Section III. Applications of real-time data are described in Section IV. Future directions and concluding remarks are made in Section V and Section VI.

\section{Remote Monitoring System OVERVIEW}

Microgrids installed in rural communities typically consist of power sources, such as photovoltaic (PV) panels and generation sets; station batteries; control equipment; inverters; and distribution systems, as shown in Fig. 1. The purpose of the RM system is to measure technical quantities from the microgrid and transmit them to a remote server in a timely manner for monitoring and analysis. Remote monitoring can be accomplished through a wide range of approaches, from simple logbooks to RM-integrated off-the-shelf components and bespoke systems.

\section{A. System Architecture}

The main components and architecture of an RM system installed at a microgrid energy kiosk in Zambia are shown in Fig. 2. The data logger samples analog and digital signals sent from current transducers (CTs), a voltage divider network, and digital pulses from energy meters. The data logger transmits the present analog readings, along with the pulse counts, once per minute to a remote server through the cellular network. 


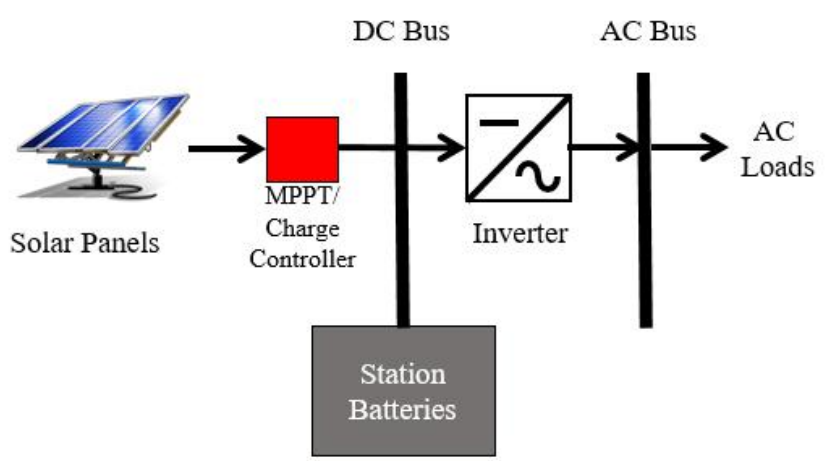

Fig. 1. Schematic of a common microgrid.

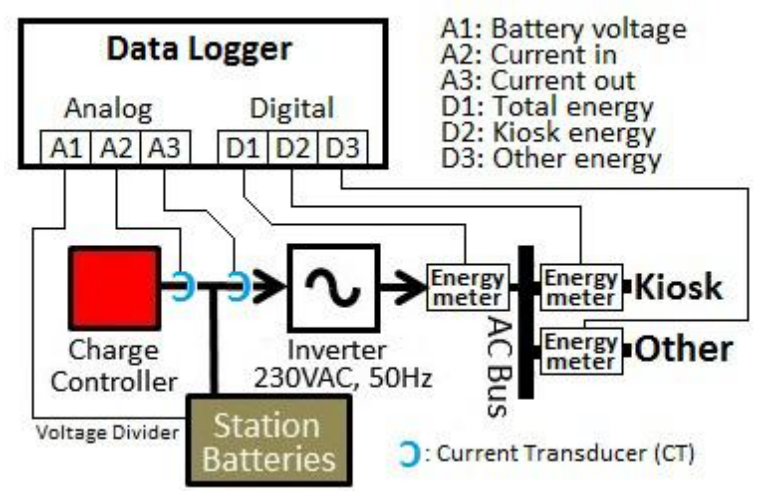

Fig. 2. Main components of a remote monitoring system.

\section{B. Data Logger}

The data logger is a small computing system with generalpurpose input/output (GPIO) pins. Data loggers are designed to process, record, and/or transmit voltage or current values measured by their GPIO pins. Data loggers can also offer supervisory control via built-in relays. These relays can be configured to control other hardware based on processed data input, or by user command. Due to the use of TTL, data loggers are typically designed in a way that their GPIO pins can measure values from 0-5 volts. Because of this, values often need to be scaled down before input to the data logger.

\section{Sensors}

Sensors convert physical quantities to information signals that are useable by the data logger. It is not necessary to use a sensor to measure all branch currents and node voltages, as some may not be of interest, and others can be obtained through application of, for example, Kirchhoff's Current Law. For the microgrid in Fig. 2, two DC CTs, a voltage divider and a set of energy meters is all that is needed to track the health, production and consumption of the system.

Due to the typically large branch currents in microrids, series measurement of current is not practical. Rather, using external transducers is a viable option. DC CTs are low-costapproximately US\$15-options for measured DC branch currents. DC CTs rely on the Hall Effect principle to sense current. Some models can be fitted around cables without interrupting service. However, due to the use of the Hall Effect, the CTs need an external DC power source.
A resistive voltage divider can be used to scale the DC bus voltage to an appropriate level for the data logger. Power and energy can often be computed from branch current and node voltage measurements. However, energy meters can be useful, particularly on the AC side of the microgrid. The meters can be installed downstream of AC breakers. They commonly output a digital pulse each time a threshold quantity of energy has been consumed by the circuit, for example, one pulse per watthour. The pulses can be counted to show total energy production, or differenced and divided by time to infer power consumption. Energy meters can be purchased for approximately US\$15.

\section{Communication}

Due to the rural nature of these microgrid installations, landline internet access is typically not available. If the installation is within range of a local cell phone tower, the service provider's SIM card can be used to connect to the internet. For a data logger to facilitate this type of internet access it must have a modem that can communicate over the local band, typically $900 \mathrm{MHz}$ or $1800 \mathrm{MHz}$, and have a SIM card with an active plan with internet permissions.

\section{E. Server}

Modern computers are powerful enough to be a useful server. A server remotely provides services to other computers. Operating systems built on open-source free kernels (e.g. Linux, GNU, or Berkeley's BSD) can be composed of entirely open-source free software and are easily configured to run services (e.g. web servers, secured remote access and/or data receiving and processing services). At minimum the server needs to listen to the port that the data logger is transmitting to and record all the data it receives. To facilitate a useful dashboard the server also needs to store the data in a structured way that can be easily and quickly accessed, typically done with free database software (e.g. MySQL or SQLite).

To implement data protection, and improve reliability of data access, multiple computers should be utilized. A server farm that provides cloud computing services serves this purpose very well due to reduced costs via specialization.

\section{F. Dashboard}

The dashboard, also known as the "front-end" or "portal" is at minimum an html file that defines the layout of the web page, as well as some server side scripts that the html file references. Done in this way, the scripts give the user the ability to query the data. There are many web server modules and interfaces, such as Common Gateway Interface (CGI) or the Python Web Server Gateway Interface (WSGI), that allow the webserver to dynamically build the html file that it returns to the user. This offers much more useful and flexible dashboards and helps facilitate displaying graphical data that has been processed in some way. There are several software packages available to configure the server's database, web server and dashboard, for numeric time-series data.

\section{G. Integrated Systems}

Several off-the-shelf charge controllers and inverters now include integrated data acquisition systems [8,9]. Pre-packaged 
integrated monitoring, management and control products and services are also available [10].

The products and services differ by what quantities they are capable of measuring, their means to communicate data and protocols used. Some systems are designed for use in PV-only systems, whereas others offer a broader range of quantities they can measure. Communication and storage methods also very, including telephone line, Ethernet, analog and GSM modems, or in an SD card. Some products use an open Modicon Modbus transmission protocol which is useful for integration with other technologies. The services offered vary. Some provide complete dashboards and are able to identify of underperforming strings as well as performance ratio for maximum system output. Some products can communicate with energy meters through built-in Ethernet, RS-232 and RS485 ports.

\section{REMOTE MONITORING SYSTEM CONSIDERATIONS}

In deciding whether or not to install a RM system, the benefits of improved technical sustainability must be compared to the upfront and on-going costs. In general, the cost of RM does not scale proportionally with the capacity size of the system, so that installing RM for large systems is more attractive than for small systems. Other factors to include in deciding to install an RM system are the organizational ability to maintain the RM system and to apply the data in a beneficial manner. Once the it has been decided to install an RM system, the designer must consider several aspects, as discussed next.

\section{A. Quantities to Measure}

Determining which quantities to measure is a critical design decision. Data loggers come with a limited number of analog and digital channels, and the cost of sensors may limit the number of quantities that can be measured. Prioritization is often required.

We have found that the single measurement that provides the most insight into the "health" of the system, if properly interpreted, is the terminal voltage of the station battery [6]. Measuring load current or power at the furthest downstream point allows the general functionality of the upstream components to be verified - if the load is being served, then the battery, generation sources, inverter, circuit breakers, and other upstream components have at least some level of functionality. The branch current from generation sources such as solar panels is also useful. Many renewable resources follow somewhat predictable patterns. Monitoring the current for evidence of these patterns is a simple way of diagnosing whether the generation source is behaving as expected.

Measuring the branch currents at the DC bus as well as the battery terminal voltage allows the power from the sources, into and out of the battery and to the load to be computed. This provides a complete picture of the basic operation of the system, and can be extremely useful in monitoring the performance of the microgrid.

Eliminating measurement redundancy reduces cost, but if the cost saving is incremental, then, for example, measuring all branch currents on the DC bus allows the user to do "sanity" checks on the data, ensuring KCL is obeyed. If not, then there is a measurement or other error.

Measuring quantities such as temperature, wind speed, and solar insolation can be insightful as well. For example, the data be used to distinguish between whether low wind turbine energy production is due to low wind speeds or turbine malfunction. However, these measurements often require specialized sensors and mounting, which adds to the cost of the RM system.

The sample rate of the measurements affects how the data can be used. High resolution sampling is more insightful than low resolution. We have found that sampling periods between one and 15 minutes to be useful, practical and easy to implement.

\section{B. Data Transmission and Access}

Local conditions will often affect the choice of data communication technology to employ in the RM system. Local coverage of GPRS, $2 \mathrm{G}, 3 \mathrm{G}$ or $4 \mathrm{G}$ networks varies, and in some places the connection quality is minimal. Online resources are available to better estimate the coverage within a country, but our experience is that signal strength and availability is best measured locally.

The data broadcast rate is a critical decision. The broadcast rate can be different from the sampling rate, for example if the RM system collects multiple samples across time and sends them periodically in bulk. This reduces the transmission data charges incurred that often accompanies RM systems. However, the ability to monitor the system in real-time decreases.

\section{Other Considerations}

Some RM systems require a SIM card with a local network provider. In many countries, data and SMS credit is pre-paid and expires after 90 days, making it challenging to maintain credit over time. Some providers now allow credit to be added on-line. Data rates may also vary. A RM system in Zambia broadcasting minutely data over the MTN network typically consumes US\$5 per month.

Battery back-up requirements of the RM system should also be considered. Should the microgrid lose power, it is often desirable for the RM system to continue to function, as this will assist in restoring the operation of the microgrid. We suggest a minimum of four hours of internal RM battery storage.

\section{REMOTE MONITORING DATA APPLICATIONS}

In the following, we outline several applications of RM data, using illustrative examples from actual systems.

\section{A. Performance Verification}

Tracking the performance of a microgrid against target metrics or outcomes is an important use of RM data. Donors and current or perspective investors increasingly require hard data on technical performance. For microgrids, an important performance indicator is the energy consumed by the load. Energy consumption as a metric accounts for losses and is perhaps more closely related to the developmental or revenue 
goals of the microgrid. A plot of energy consumption for a $2 \mathrm{~kW}$ PV system in Zambia is shown in Fig. 3.

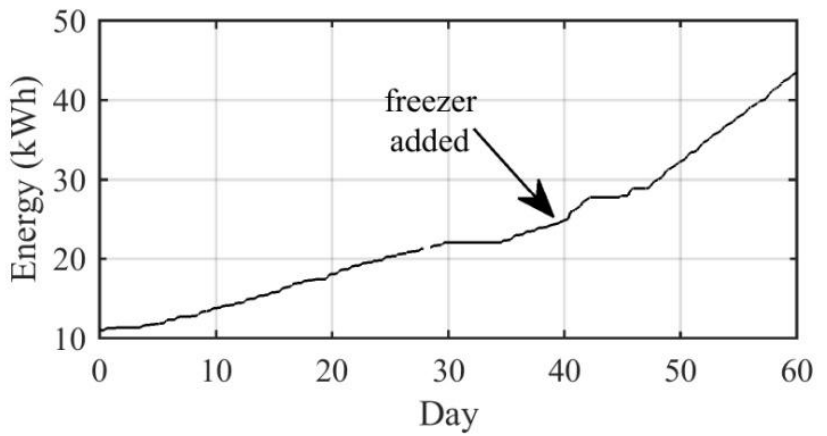

Fig. 3. Energy consumption data is useful for validating performance and for making energy management decisions, such as adding load if the system is under-utilized.

Should energy consumptions not be measured by the RM system, then the energy input to the inverter is the next preferred measurement point, although it includes inverter losses. Using the energy produced by the source as metric is a less attractive alternative since all system losses are included in this metric, which can inflate the value by 40 percent [6].

\section{B. Operation Monitoring}

Receiving real-time data allows the operation of the microgrid to be manually or automatically monitored. Manual interpretation of real-time microgrid time-series data requires nuance. Several techniques are discussed in [6]. Interpreting the data requires understanding of controller algorithms, setpoints and interaction.

Using RM data to determine if the batteries are fully charged on a daily basis is a particularly useful application. As discussed in [6], attempting to estimate battery state of charge from battery's terminal voltage is error-prone as the internal impedance and charging/discharging current can greatly affect the terminal voltage. A better approach is to monitor the terminal voltage to see if the absorption stage set-point of the charger controller is reached, and how long it remains at that set-point. For example, the data in Fig. 4, which comes from a hybrid PV/wind microgrid in Kenya, reaches the absorption set point during the first two days, but it does not become fully charged during the next several days. If this continues, the lifetime of the battery can be reduced prematurely. The operator was instructed to reduce load, allowing the battery state of charge to recover, which it does on day 10.

Other monitoring applications include: verifying the control scheme is operating as planned; determining if major components are operating properly; and determining if the operator is following instructions.

\section{Energy Management}

A noted challenge in microgrid design in the context of LEDCs, is the accurate estimation of the demand. In addition, behavior patterns change over time. An important application

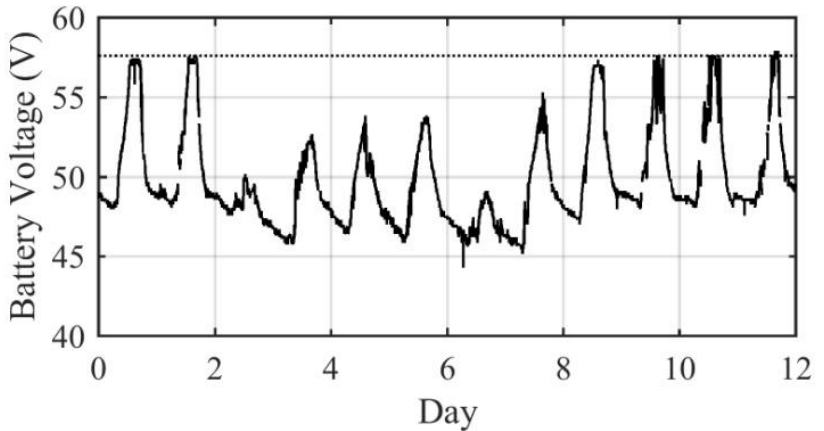

Fig. 4. Battery voltage over a 12-day period from a microgrid in Kenya. The dashed line is the charge controller absorption stage set point.

of data is to determine if the installed microgrid is being overor under-utilized. Over-utilization can result in pre-mature failure due to prolonged battery low state of charge and upset customers due to low reliability. Under-utilized systems can be viewed as a wasted investment. Over- and under-utilization can often be corrected by interventions such as adjusting tariffs, installing more energy efficient appliances or adding/removing energy intensive services. For example, after reviewing the RM data for the microgrid in Zambia, it was determined that the system was under-utilized. A freezer was added to one of the circuits to sell refrigerated goods. The effect of the freezer is evident in the accelerated rate of energy consumption in Fig. 3.

\section{Billing/Customer service}

Many new microgrids act as micro-utilities, where electricity is supplied to customers at an agreed upon tariff. In this context, the energy used by the customer is the basis for the tariff, which necessitates the energy consumption to be metered. So-called "smart meters" measure and periodically report energy use values via a cellular network to the cloud. Business management systems track customer payments and can send signals to disconnect customers once their pre-paid credit has expired.

\section{E. Failure Analysis}

Despite best efforts, microgrids can have their power supply interrupted. RM data can offer a way for the cause of the failure to be identified, much in the same way as a "black box" in an airplane. For this application, high temporal resolution data is needed-preferably at least once per minute - depending on the fault condition. Low resolution data may also be useful, for example to determine if the inverter is outputting the proper voltage.

\section{F. Design}

Accurate load estimation is critical in microgrid design but has plagued designers [3]. Long term analysis of RM data can be used to check the accuracy of load estimation. Collecting load data of different customer classes, such as shown in Fig. 6 can improve future microgrid designs. 




Fig. 6. Load profile of a microgrid-connected home with mean (solild) and $25^{\text {th }}$ and $75^{\text {th }}$ percentile (dashed) shown.

\section{FUTURE DIRECTIONS}

Exploration of real-time off-grid data sets from LEDCs is being led within the IEEE PES Working Group Sustainable Energy Systems for Developing Communities (SESDC) [11]. The Working Group's overall aim is to raise awareness about access to energy systems, including electricity and the related issues in developing communities around the world. In 2015 the Working Group formed the Energy Use in Off-grid Applications Task Force, to further study this area of research.

Specific research outputs have been identified and include modeling the usage profiles of various types of loads, better understanding seasonality and other trends including load growth, determination of predictors of energy use, evaluation of the accuracy of demand estimation methodologies, links energy use and sustainability, calculation of real-world inefficiencies of system and sub-components, and quantifying the reliability versus cost tradeoffs of design approaches.

With growing availability of the technology and data sets, there is a distinct opportunity to inform future delivery of offgrid systems. To maximize the contributions that the research community can make, an open data archive is needed that accumulates, stores and responsibly allows access to conduct this research. Data sets would include different system sizes from small solar home systems to village-scale microgridsbut would maintain a common data architecture for analysis. Further work is needed to define the hardware, software, and protocol requirements suitable for this purpose.

RM technology products and services in LEDCs for energy access applications are relatively immature despite the existence of comprehensive functionality of similar products (namely, SCADA) in industrialized countries. Yet the market is gathering attention as suppliers warm to the opportunities. Product design in this space can be informed by thorough analysis of the usage profiles, requirements, and exploration of potential synergies of combining energy and internet access. 'Information' poverty in this context, like energy poverty, is cross-cutting and equally critical. Bridging the information gap has the potential for both commercial opportunities, though the provision of knowledge services, and achieving socioeconomic benefits such as energy access and improved education. Enabling and extending RM, as the core component of this functionality can therefore be considered strategic in nature.

Finally, there is a need to develop a better understanding of micro-level energy use trends to underpin national and international planning. Long-term models of energy use, such as the WEM model used by the IEA [12], involve broad assumptions on the progression through the energy ladderseminal studies such as [13] could be extended to lowerincome cohorts in developing countries. For off-grid users in developing countries, this analysis remains incomplete. Improving the results of such models, which are extremely influential for policy makers and practitioners alike, could likewise improve the coordination of investments in the sector.

\section{CONCLUSIONS}

Remote monitoring (RM) systems are increasing in popularity in rural microgrids in less economically developed countries. This paper outlined the key components of the RM systems, important considerations in devising such systems and described several applications of RM system-collected data.

\section{REFERENCES}

[1] International Energy Agency, "World energy outlook 2011." 2011.

[2] H. Louie, et al., "Eternal Light: Ingredients for Sustainable Off-Grid Energy Development," Power \& Energy Magazine, vol 12, no. 3, pp. 70-78, DOI: 10.1109/MPE.2014.2317093, Jul./Aug. 2014.

[3] L. Flowers, "Lessons learned-NREL Village Power Program," Tech. Rep. WE805010, National Renewable Energy Laboratory, Jul. 1998.

[4] N. Cross, C. Gaunt, "Application of rural residential hourly load curves in energy modelling," in Proc. IEEE Power Tech Conference, Vol. 3, Bologna, Italy, 2003, pp. 1-4, DOI:10.1109/PTC.2003.1304492.

[5] P. Dauenhauer and H. Louie, "System Usage Trends for Off-grid Renewable Energy Users in Developing Communities," in Proc. of $4^{\text {th }}$ Symposium on Small PV Applications, Muchen, Germany, Jun. 2015.

[6] H. Louie, "Operational Analysis of Hybrid Solar/Wind Microgrids Using Measured Data," Energy for Sustainable Development, vol. 31, pp. 108-117, Feb. 2016.

[7] K. Weber, S. Kennedy, P. Armstrong, End-use load monitoring of a micro hydroelectric-powered community microgrid: a case study in rural Malaysia, in Proc. Innovating Energy Access for Remote Areas, Berkeley, CA, pp. 23-25, 2014.

[8] Steca, "TarCom datalogger fo Tarom system manager," Installation and Operation Manual, [Online]. Available: http://cranebg.com/files/steca_tarcom_manual_en.pdf. Accessed Feb. 2016.

[9] N. Wolfe, "An open--source monitoring system for remote solar power applications", [Online]. Available: solar-monitoringproject.googlecode.com/files/FinalReport.pdf. Accessed Feb. 2016.

[10] M. Wollny and M. Hermes, "AC coupled hybrid systems and minigrids," ANZSES Solar, 2007.

[11] IEEE PES Working Group on Sustainable Energy Systems for Developing Countries. [Online]. Available: http://sites.ieee.org/pessesdc/. Accessed Feb. 2016.

[12] International Energy Agency, "World Energy Model", [Online]. Available: http://www.worldenergyoutlook.org/weomodel/ Acessed Feb. 2016.

[13] C. Wolfram, O. Shelef and P. Gertler. "How Will Energy Demand Develop in the Developing World?," Journal of Economic Perspectives, vol. 26(1), pp. 119-38, 2012. 